International Journal of Biomedicine | June 2019 - Volume 9, Issue Suppl_1: Abstracts From the Second Russian International Conference "Cryo-electron microscopy 2019: achievements and prospects"

ORAL ABSTRACT PRESENTATIONS

SESSION TITLE: APPLICATIONS OF CRYO-EM IN MEDICINE

DOI: 10.21103/IJBM.9.Suppl_1.OR13

\title{
Abstract OR-13: Functional and Structural Studies on Human ASCT2 Transporter - a Promising Drug Target
}

\author{
Albert Guskov ${ }^{1,2}$, Alisa Garaeva ${ }^{1}$, Cornelius Gati $^{3}$, Cristina Paulino $^{1}$, Dirk Slotboom ${ }^{1}$ \\ ${ }^{1}$ Groningen Biomolecular Sciences and Biotechnology Institute, University of Groningen, the \\ Netherlands; ${ }^{2}$ Moscow Institute of Physics and Technology, Dolgoprudny, Russia; ${ }^{3}$ Stanford University, \\ Department of Structural Biology, Stanford, CA, USA
}

Background: ASCT2 protein belongs to Solute Carrier Family 1 (SLC1A) transporters and is a promising target for the development of anticancer and antiretroviral drugs. It is upregulated and linked to poor survival in melanoma, lung, breast, prostate, pancreatic, thyroid and colon cancer and serves as a receptor for simian retrovirus 4 , feline endogenous virus, human endogenous retrovirus type $\mathrm{W}$ and baboon M7 endogenous virus. However, there is a lack of structural and functional characterization of this protein.

Methods: We used single particle cryo-electron microscopy to obtain three-dimensional models of ASCT2 in two distinct conformations - inward-occluded and inward-open at resolutions of 3.85 and $3.6 \AA$ respectively.

Results: The structural comparison of ASCT2 in inward-occluded and inward-open states revealed the different positions of mobile HP2 loop. It has moved $8 \AA$ away from the substrate binding site towards the scaffold domain, thus allowing direct access to the binding site from the cytoplasm. Apart from that the conformation of the transport domain is very similar in both structures, however in the inward-open state it is slightly further tilted towards the cytoplasm, away from the scaffold domain. The analysis of densities revealed possible positions of several lipids/cholesterol molecules. The most peculiar position is at the HP1/HP2 interface, where a lipid could play a regulatory role of the loop closure / opening. Other positions are between the scaffold and transport domains; intriguingly one such position coincides well with the previously described allosteric inhibitor binding site in EAAT1 transporter.

Conclusion: The reported structures of an inward-open state of ASCT2 transporter confirmed an earlier hypothesis of one-gate elevator mechanism, which is different from any other secondary-active transport mechanism described to date. The observed several lipid densities in the vicinity of the binding site and around scaffold might represent potential inhibitor sites for rational drug design.

Key Words: membrane proteins $\bullet$ cryo-EM $\bullet$ SLC1A transporters $\bullet$ cancer $\bullet$ retroviruses 
International Journal of Biomedicine. 2019;9 Suppl 1: S11. doi: 10.21103/JJBM.9.Suppl_1.OR13 (C)2019 International Medical Research and Development Corporation 